\title{
ADRs and Children: Knowledge and Methodological Standard in PASS (Post Authorization Safety Studies)
}

\author{
Ettore Napoleone and Cristiana Scasserra \\ FP-MCRN (Family Pediatricians - Medicines for Children Research Network), 86100 Campobasso, Italy
}

\begin{abstract}
PhV (pharmacovigilance) knowledge has the outcome of being inadequate in particular due to the lack of instruction. Pediatricians have to face the realization that over-the-counter medicines increments the peril of ADRs (adverse drug reactions) has become a public health concern. The purpose of this article is to disseminate knowledge of the PhV and to highlight the cultural and organizational difficulties for its implementation. The objectives of promoting the organization of specific training courses and research projects aimed at: (1) to foster the culture of iatrogenic disease in pediatrics; (2) to improve the appropriateness of prescribing drugs in children; (3) to encourage spontaneous reporting of ADRs in children; (4) to involve Pediatricians in PASS (post-authorization safety studies) according to GCP, GVP and ENCEPP Code of Conduct. An up to date proposal of PhV, a procedure of preparation improves the productivity of creating novel informative reports which can be utilized for a benefit/risk scrutiny making strides in medicine prescription. In this context, FP-MCRN (family paediatricians-medicines for children research network) established with the aim of developing competence, infrastructure and education for paediatric clinical trials, plays a crucial role in paediatric $\mathrm{PhV}$, through an improvement of $\mathrm{PhV}$ training, a correct research methodology and very strong relationship with the families. The initial necessity is cultural, implementing culture of iatrogenic illnesses and a watchful evaluation of the importance of PASS by FPs (family pediatricians). Participation in clinical trials that generates a precise database administered by FPs together with follow-up outcomes becomes relevant and vital for a scrupulous and accurate assessment of ADRs. PASS can foresee on one hand training and information regarding the proper use of drugs in children and possible iatrogenic pathologies caused by their improper use, the other to constitute a territorial survey in the prescriptive appropriateness and safety of pediatric drugs aimed at evaluating the risk-benefit balance on usage.
\end{abstract}

Key words: Children, adverse drug reactions, off-label, pharmacovigilance, post-marketing clinical studies.

\section{Introduction}

The risk of ADRs (adverse drug reactions) in children has become a public health concern. In paediatric populations the problem is enhanced by the fact that ADRs are rarely represented in RCCTs (randomized controlled clinical trials). The differences such as pharmacokinetics, pharmacodynamics, epidemiology and co-medication between children and adults in general as well as between children of different age groups, the adverse event and ADR profiles may be different in children and adults. In addition most genetic and rare disease has a childhood onset which adds to the diversity of this population from the adult one [1-4].

Paediatricians should be aware that the use of off

Corresponding author: Ettore Napoleone, Pediatrician, President of FP-MCRN, research fields: pediatric drugs. label drugs increases the risk of adverse reactions and they should also try to pay much more attention when they administer drug therapies to their patient [1-8]. To address the issue of off-label and unlicensed prescribing in children, European regulations were issued with the scope of obliging pharmaceutical companies willing to register new compounds, or indications and formulations, for paediatric use, to submit “paediatric investigation plans” from January 2007 [9].

In Italy regulatory authorities have increased efforts to enhance awareness of health professionals about the importance of their reporting of ADRs as a significant contribution towards the process of paediatric pharmacovigilance. PASS (post-authorization safety studies), which have been regarded as a strong characteristic to be directed towards the recovery of all 
events that occur during monitoring, give much more reliable estimates of the risk of ADRs than those resulting from spontaneous reports [10-17].

The purpose of this article is to disseminate knowledge of the $\mathrm{PhV}$ and to highlight the cultural and organizational difficulties for its implementation. The objectives are of promoting the organization of specific training courses and research projects aimed at: (1) to foster the culture of iatrogenic disease in pediatrics; (2) to improve the appropriateness of prescribing drugs in children; (3) to encourage spontaneous reporting of ADRs in children; (4) to involve Pediatricians in PASS (post-authorization safety studies) according to GCP, GVP and ENCEPP Code of Conduct.

\subsection{The FP-MCRN (Family Paediatricians-Medicines for Children Research Network)}

In this contest relevant proactive roles are being played by the FP-MCRN (family paediatricians-medicines for children research network) to develop new methodological approaches and optimise use of available resources, to enable the system to recognise issues of efficacy and safety reducing the health risks for such fragile populations for children as early as possible [1-6].

The FP-MCRN, ENCePP and Enpr-EMA member, established with the aim of developing competence, infrastructure, networking and education for paediatric clinical trials, plays a crucial role in paediatric $\mathrm{PhV}$, through an improvement of $\mathrm{PhV}$ training, a correct research methodology and very strong relationship with the families. Through specific training (training and educational courses) FP-researchers acquire in fact a correct research methodology according to GCP and GVP (module VIII of the good pharmacovigilance practices on post-authorization safety studies) standards, to the ethical Consideration and to the code of conduct of ENCePP for the conduct of clinical studies on children. FPs (family pediatricians) have a close and elective involvement with children and their families establishing a very strong and close "pediatrician-parents" and "pediatrician-child" relationship. For this reason it becomes easier for the capacity to assess and involve outpatients in clinical trials and the possibility to give more accurate information on the correct use of pediatric drugs [1-6].

\subsection{The "ENCePP Study Seal”}

To recognize and study following the ENCePP principles of methodological standards, transparency and independence, the "ENCePP study seal" has been introduced. Studies have to be conducted in accordance with the following principles that may qualify for the "ENCePP study seal": (1) the highest possible level of transparency regarding the use of methodological standards in the study protocol; (2) the contractual arrangements between investigators and the study funder clearly define the research assignment and address critical areas of interaction, remuneration, protocol agreement, analysis of results and publication of results.

The study is registered in the EU PAS Register (ENCePP E-Register of studies) prior to its start, thereby making publicly available information on the research process; the study results (synopsis or manuscript) are published in the EU PAS Register in line with agreed timelines.

In February 15, 2016 a FP-MCRN REDS (respiratory drugs survey) study: active surveillance of respiratory drugs, especially IS (inhaled steroids) in children (AIFA PhV CALL 2010-2011) had awarded with the ENCePP study seal according to principles of methodological standards, transparency and independence [18].

On 383 pediatric studies in the EU PAS Register only 26 (6.7\%) had awarded with the ENCePP study seal. The REDS Study was one of these 26 pediatric studies. This shows that the FP-MCRN has acquired the skills and the experience to build high quality clinical studies, with methodology and scientific rigor.

REDS Study was a PASS conducted in the territory led by family paediatricians that can on the one hand 
predict the FPs training courses and information on families regarding the use of respiratory medication, especially inhaled steroids and any iatrogenic condition caused by their improper use, on the other hand may constitute an active territorial survey on prescriptive appropriateness and safety of these drugs in children aimed at evaluating the risk-benefit balance on usage. This study will enable a wide spread to the FPs GL (guidelines) reference and implement skills (FPs training) for a correct diagnosis, to a viable therapy and closely follow up of pathologies that require the use of these drugs in all children from 0 to 14 years regarding their clinical seriousness. Finally, the study will encourage closer synergy between FPs and families through better and more accurate information given to families on the use of respiratory drugs and in particular of IS will lead to a reduction of the "do it yourself" and self-prescription for illnesses that do not include their use in GL and will certainly lead to a reduction on working days lost by parents and of any hospitalizations (reducing inappropriate admissions) [18].

\section{Aim}

The purpose of this article is to disseminate knowledge of the pharmacovigilance and to highlight the cultural and organizational difficulties for its implementation to create, through the magazine, a debate on the issues that may arise.

Paediatricians should be aware that the use of off label drugs increases the risk of ADRs and they should also try to pay much more attention when they administer drug therapies to their patients. The potential risk of ADRs in children has become a public health concern and regulatory agencies in Europe have acknowledged the need for urgent research efforts in this area. In particular the spontaneous reporting of suspected ADRs is an important means to promote reasonable warning signals. Epidemiological post-marketing studies, which have as a strong characteristic to be directed towards the recovery of all events that occur during monitoring, give much more reliable estimates of the risk of ADRs than those resulting from spontaneous reports. In this context, FP-MCRN (family paediatricians-medicines for children research network) established with the aim of developing competence, infrastructure and education for paediatric clinical trials, plays a crucial role through an improvement of pharmacovigilance academic training, a correct research methodology and very strong relationship with the families

\section{Materials and Methods}

In 2013, we performed a survey to evaluate FPs knowledge, feeling and compliance in ADR reporting. A total of 552 FPs evenly distributed throughout the Italian territory provided a feedback to the survey. $\mathrm{PhV}$ (knowledge of pharmacovigilance) resulted to be poor, mainly due to the absence of adequate training in academy; despite this, the majority of FPs declared to be interested to $\mathrm{PhV}$ and was aware of its positive impact on their clinical practice. Most of FPs considered the academic preparation in $\mathrm{PhV}$ "insufficient" (76.5\%) and only $1 \%$ of the responders defined their preparation as "exhaustive". However, 92\% of FPs had the perception that PhV can improve their patients care and $78 \%$ would be willing to participate in specific training, if made available. This study provided the first Italian survey on paediatric PhV. It highlights that FPs are aware of PhV importance in their clinical practice while recognizing an insufficient education on it. Considering these observations, we believe that an improvement of $\mathrm{PhV}$ academic training, continuous training and information at the territorial level, possibly coordinated at the national and transnational levels may generate a better compliance and thus optimization of ADRs reporting [19].

\subsection{Spontaneous Reporting}

In Italy, the activity of spontaneous reporting in children is very low: in recent years, paediatric data-reporting has stood at around 1 to 1.6 percent, 
compared with $8 \%$ of total reporting. Reasons may be different: (1) a non spread of a iatrogenic disease culture over the years' existence; (2) people don't understood the real benefits that the spontaneous reporting system can create for the community in terms of reducing the risks of patients and saving resources $[1,2,19]$.

Family Pediatricians case reports mainly involve vaccines followed by frequency reports of pharmaceuticals used by children: antibiotics, antipyretics, inhaled corticosteroids, specific products for colds and some drugs belonging to the class of drugs on the gastrointestinal tract and metabolism. Most of the ADRs, observed in pediatric prospective studies, mainly effect the skin (rash, urticaria) and the gastrointestinal system (diarrhea, nausea and vomiting), but we can also observe systemic reactions and reactions connected to the central nervous system [1-7].

Very often a major cause of ADRs is determined by the behavior of families, when facing adverse reactions due to a non-rational use of drugs. A high percentage of the accesses to the emergency room for adverse reactions have been registered, after an ingestion of an overdose of medication. The main causes of overdoses are: accidental ingestion of the drug for the lack of adult control, defective or inadequate packaging (for example, the lack of safety lock systems), and the error in the preparation/dilution of a more concentrated drug [1-3].

We developed a pilot $\mathrm{PhV}$ project named "MEAP (monitoring of the adverse effects in paediatric population)", a design to enhance high quality spontaneous reporting of ADRs in children by both hospital and family paediatricians [2].

The Italian pharmacological society and family paediatricians decided to act in concert and have generated a "recommendations for quality pediatric pharmacovigilance" guideline, which is currently being distributed to all family paediatricians [2].

The MEAP project demonstrates that an approach of pharmacoviglinace accompanied by continuous training, sharing of results and easy to fill reporting forms, enhances the effectiveness of generating new informative data which can be exploited for benefit/risk analysis, improving drug prescriptions and ameliorating the current regulation, thus ultimately impacting on paediatric patient safety. Nevertheless much more efforts is needed to guarantee that children will receive the correct medications, appropriate to their clinical conditions [2].

\subsection{PASS (Post-Authorization Safety Studies)}

When the drug is used in clinical practice in large unselected populations, PAS Studies, according to GCP, GVP and ENCePP code of conduct, are useful as they find their major confirmation in recalling all the events that occur during monitoring, with estimates of incidence of ADRs that cannot be obtained by spontaneous reports. In these studies a significant role can be played by the FP with the participation to active pharmacovigilance projects [1-6].

AIFA (The Italian medicines agency) approved, for the first time ever, a project conceived, structured and implemented by the family pediatricians and presented by the Molise region, within the program of active pharmacovigilance funded through the regional funds available for the years 2008 and 2009 [20, 21].

"From the data OSMED of prescription of pharmaceuticals in which a widespread of antibiotics were calculated, stratified by age, once again there emerges an "over-prescription" of these drugs, especially in the age group between 0 and 2 years. An increase in ADRs related to them is noted. It still seems obvious that inefficient use of these drugs as instructed are not always based on scientific evidence raising the problem of antibiotics-strength and quality of the therapy. Hence the need for a research project, Phase $\mathrm{IV}$, is managed and coordinated in full for the first time with the family pediatrician, that can foresee on one hand training and information regarding the proper use of antibiotics in children and possible iatrogenic pathologies caused by their improper use, the other to 
constitute a territorial survey in the prescriptive appropriateness and safety of these drugs in children aimed at evaluating the risk-benefit balance on usage. The study wanted to firstly exceed the limitations of the data OSMED which they see as an impossibility to track the diagnosis that is the basis of the prescription, and of a correct valuation of prescriptions in the various pediatric age groups (especially 0-2 years )" [21-23].

The study conducted by 37 Family Pediatricians consisted of three phases: (1) in the first phase (retrospective-year 2013) we assessed the prevalence of antibiotic prescriptions in the 0 to 2 years age group, the type of antibiotic used and any ADRs; (2) the second phase (2014) was that of training/information to the FPs and families on prescriptive appropriateness and proper use; (3) in the third phase (year 2015) the prevalence of prescriptions has been reassessed and the type of antibiotic used and the possible ADRs (after the training phase). These data had been compared to the data of prevalence of 2013 [21-23].

The study showed, in the first phase (2013) that the prevalence of antibiotics prescribed by $37 \mathrm{FP}$ from $0-2$ years old (4060 children), was 83\% (number of children with at least one prescription: 3339) with a number of prescriptions of 7114 (number of items prescribed: 8367) (Fig. 1, Table 1).

After training, the year 2015 detected a 56\% prevalence (number of children 4116, number of children treated 2327) with a significant decrease (-27\%) compared to 83\% in 2013. In addition there has been a reduction of 2938 prescriptions (number of prescriptions 4176) and a reduction of 2975 prescribed items (number of items prescribed 5392) with savings

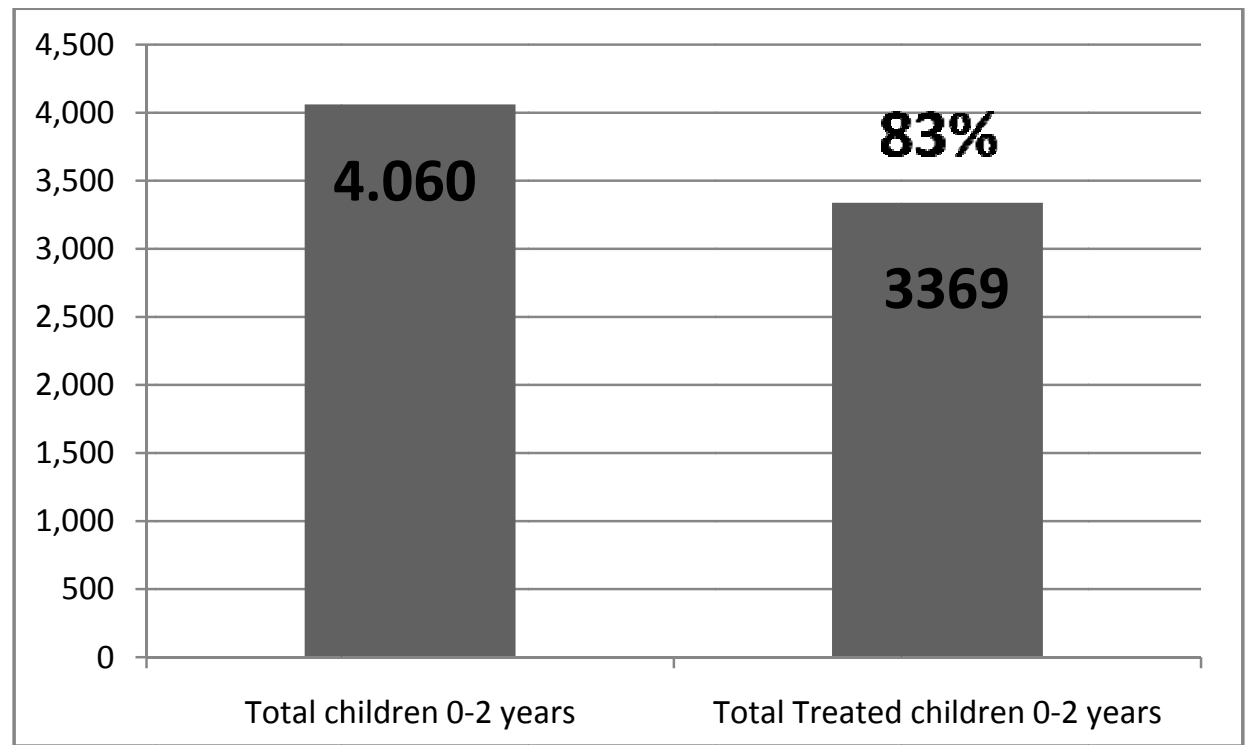

Fig. 1 The prevalence of the use of the antibiotics in 2013 was $83 \%$.

Table 1 Prescription indicators and expenditures 2013.

\begin{tabular}{llll}
\hline Zone/District & Prescriptions & Pieces & Expenditures (euro) \\
\hline Agnone & 104 & 109 & 931.85 \\
Isernia & 1,361 & 1,766 & $12,027.97$ \\
Venafro & 543 & 749 & $4,412.98$ \\
Bojano & 775 & 860 & $5,268.95$ \\
Campobasso & 1,925 & 2,259 & $15,258.13$ \\
Larino & 587 & 653 & $5,318.99$ \\
Termoli & 1,819 & 1,971 & $17,731.29$ \\
Total & 7,114 & 8,367 & $60,950.16$ \\
\hline
\end{tabular}


PASS (Post Authorization Safety Studies)

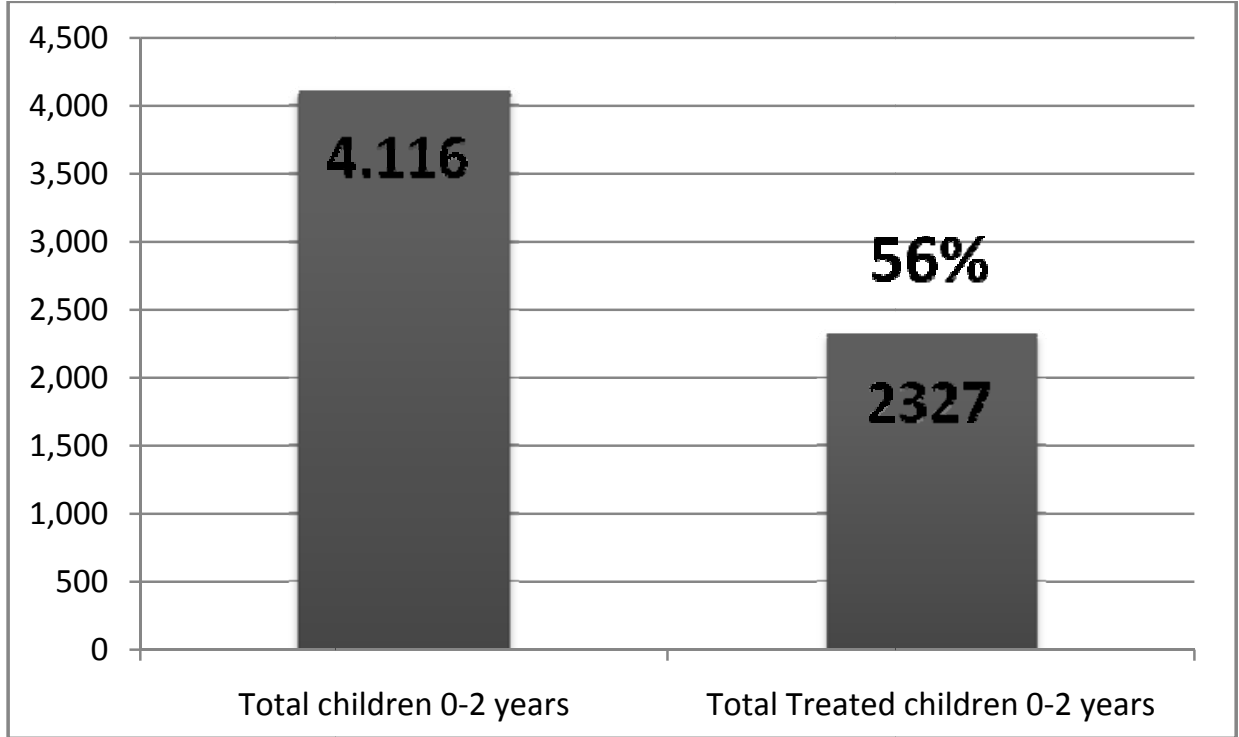

Fig. 2 The prevalence of the use of the antibiotics in 2013 was $56 \%$.

Table 2 Prescription indicators and expenditures 2015.

\begin{tabular}{lllll}
\hline District & Family Pediatricians & Total prescriptions & Total pieces & Expenditure (euro) \\
\hline Agnone & 1 & 76 & 98 & $1,175.85$ \\
Isernia & 6 & 880 & 980 & $8,158.59$ \\
Venafro & 4 & 378 & 595 & $3,292.38$ \\
Bojano & 4 & 532 & 768 & $5,119.97$ \\
Campobasso & 11 & 986 & 1,286 & $10,365.57$ \\
Larino & 3 & 348 & 523 & $3,762.72$ \\
Termoli & 8 & 976 & 1,142 & $10,220.85$ \\
Total & 37 & 4,176 & 5,392 & $42,095.93$ \\
\hline
\end{tabular}

of $€$ 18,854.23 (60950.15 EUR in 2013 and 42095.92 EUR in 2015) (Fig. 2, Table 2).

Finally there was also an improvement in the appropriateness of prescriptions according to the Guide lines: Amoxicillin (38\%), followed by Amoxi/clavulanate (29.3\%), Macrolides (16.3\%), and Cephalosporins (15.2\%). There were no ADRs in the two reference years [21-23].

\section{Discussion}

In light of the results before mentioned, there was a clear necessity to undertake an information campaign on the appropriate use of this class of medication, either through the organization of training/information events, or through the dissemination of national and international guidelines on the correct antibiotic use in therapy.
As it is known, in fact, an inappropriate use of antibiotics is a potential risk not only for individual health, with an increase in exposure to the risk of adverse reactions, but also for public health resulting in development of resistance, and the problem of costs to the National Health Service.

Among the main unjustified use of antibiotics, highlighted one is the fact of the high use of this class of medication for the treatment of colds, flu and other respiratory infections, which for $80 \%$ of cases are due to viral and not bacterial, especially in the 0 to 2 years age group, and therefore antibiotics are not effective for treating them.

The high prescription of antibiotics in the pediatric population for childhood respiratory diseases such as strep throat, acute cough, cold, mainly caused by viruses is a recognized indicator of inappropriate 
prescribing. It then proceeded to give relief to these aspects of inappropriateness prescriptive disseminating the main aspects of national and international guidelines for paediatricians. In particular, it was forwarded, as shown in the international guidelines (NICE 2008-2015), in the case of a child with a sore throat, that though there are other signs of infection concomitant respiratory tract (nasal discharge, cough) it is certainly a viral disease [20-23].

The event has paid particular attention to the importance of promoting research and pharmacological trial culture, by building an even higher skill aimed especially at protecting the most vulnerable one such as the pediatric population, and its attention to the culture of iatrogenic disease for a careful analysis of the risk/benefit ratio of medication for use in children. Also pediatrician colleagues were stimulated to major reporting of adverse reactions in children.

The aims of the project were illustrated and announced that first the data of the pediatric antibiotic prescriptions promot awareness on appropriateness of prescription through the communication of the above mentioned national and international guidelines.

Finally it highlights the information/training work done by family pediatricians with the families of their patients to stress the concept of the proper use of medicines in children, and in particular of antibiotics in the most vulnerable age group, avoiding the "do it yourself” by families, which expose children to the risk of adverse symptoms.

Overall, the importance of proper training is clear for paediatrician's appropriateness of prescribing antibiotics for this vulnerable class of children, correct information for families on knowledge and experience of pediatric medication and the importance of always consulting the pediatrician before "misuse" of medication that do not resolve the clinical solution but rather expose these young patients at risk [21-23].

\section{Conclusions}

Using a proactive approach means to organize family paediatricians in order to anticipate a possible identification of safety problems, and this reducing community risks.

A future path to follow in pediatrics is undoubtedly towards the synergy and coordination of all components of pediatrics (universities, hospitals, territories) for the organization of research projects and post-marketing programs. The starting point must necessarily be cultural: an implementation of the culture of iatrogenic disease and a careful assessment of the significance of PAS studies by family paediatricians.

From all these considerations, it is clear that we should move in different directions: the knowledge of pharmacovigilance and the involvement of FPs in PASS. There is the need to awaken FP to a culture of iatrogenic disease, to the reporting of ADRs and to the proper use of reporting forms. On the other hand, there is a need for a greater culture of research in pediatric pharmacology leading towards an increasingly pro-active PhV. Moreover, we must be able to seize the opportunities independently from scientific research institutions (AIFA, contract department, FVG regional funds) proposing safety studies on drugs for pediatric trials.

In the study on the antibiotics [21-23], after a comparison of prescriptive data of 2013 and the year 2015 after the formation of family pediatricians and the information to families on the proper use of antibiotics and of any adverse events related to their misuse, we had:

(1) A significant decline of $27 \%$ of the prevalence of prescriptions has gone from $83 \%$ (2013) to $56 \%$ (2015);

(2) A reduction in the number of prescriptions equals to 2938 and a reduction of the number of pieces prescribed equals to 2975, maintaining almost unchanged the number of assisted of $0-2$ years in the two years under review;

(3) A saving expenses of EUR 18,854.23;

(4) An improving prescriptive appropriateness 


\section{ADRs and Children: Knowledge and Methodological Standard in PASS (Post Authorization Safety Studies)}

according to guidelines: the most prescribed was Amoxicillin (38\%), followed by Amoxicillin + clavulanic acid (29.3\%), Macrolides (16.3\%), and the Cephalosporins (15.2\%).

The importance of proper training is clear for paediatrician's appropriateness of prescribing antibiotics for this vulnerable class of children, correct information for families on knowledge and experience of pediatric medication and the importance of always consulting the pediatrician before "misuse" of medication that do not resolve the clinical solution but rather expose these young patients at risk.

A significant aspect emerging from the Italian experience is also the importance of the synergy and coordination of paediatricians and pharmacologists for the organization of research projects, and clinical studies in continuous education. Such strategies will lead family paediatricians to regain possession of the research aspect of the profession through the fundamentals of science and the publishing of work about primary care.

The organization of specific training courses and research projects should meet the following objectives: (1) promote the culture of iatrogenic disease in pediatrics; (2) improve the appropriateness of prescribing drugs in children; (3) encourage spontaneous reporting of ADRs in children; (4) involve FP in PASS according to GCP, GVP and ENCEPP code of conduct.

Of course, all these supported by capacity building of FP-MCRN is central to epidemiological studies-observational and DBPC-RCT studies on medicines for children. The creation of a specific database managed and coordinated by the FP with follow-up results becomes strategic and very important for a careful and correct evaluation of ADRs. Last but not least, what must also to be mentioned is the fact that with the FP there would be fewer drop-outs in PAS studies if there is a taking charge of children and established close relationship with families.

The main target of family paediatricians should be to regain possession of the research aspect of the profession through the fundamentals of science and the production and publishing of paper work about primary care in order to guarantee greater knowledge and better decision-making.

The FP-MCRN must be aware and ready for specific training so that it can answer with authority for independent research. Quality and ethicality of research are becoming important end-points of territorial paediatrics, opportunities and challenges of family paediatricians during their education and during the sponsoring of health and prevention in the paediatric age groups.

\section{Competing Interests}

The authors declare that they have no competing interests.

\section{Authors' Contributions}

EN conceived of and drafted the manuscript. CS participated in its design and in its coordination focusing on legal and ethical issues,

\section{Acknowledgements}

No sources of funding were used to assist in the preparation of this article.

Mrs Rosanna Rea provided assistance for preparing and editing the manuscript.

\section{References}

[1] Napoleone, E. 2010. “Children and ADRs (Adverse Drug Reactions).” Ital J Pediatr 36: 4.

[2] Napoleone, E., and Radice, S. 2012. "From Pharmacovigilance to Therapy Amelioration in Paediatric Patients: the Role of the Clinical Pharmacologists and Family Paediatricians Part of a Series on Paediatric Pharmacology, Guest Edited by Gianvincenzo Zuccotti, Emilio Clementi, and Massimo Molteni.” Pharmacol Res 65 (2): 168-70

[3] Napoleone, E. 2009. "Medicines for children ADRs: from Spontaneous Reporting to Active Souveillance." Child Care, Health and Development 36 (Suppl 1): 106-7.

[4] Blake, K. V., Zaccaria, C., Domergue, F., La Mache, E., Saint-Raymond, A., and Hidalgo-Simon, A. 2014. 
"Comparison between Paediatric and Adult Suspected Adverse Drug Reactions Reported to the European Medicines Agency: Implications for Pharmacovigilance.” Paediatr Drugs 16 (4): 309-19.

[5] Clavenna, A., and Bonati, M. 2009. “ADR Epidemiology in Paediatrics.” M\&B 28: 503-4.

[6] Venegoni, M. 2009. "The Importance of Case Reports in Pharmacovigilance.” $M \& B$ 28: 501-3.

[7] Napoleone, E. 2009. “Adverse Drug Reactions and Family Paediatricians.” $M \& B$ 28: 505-6.

[8] Palmaro, A., Bissuel, R., Renaud, N., Durrieu, G., Escourrou, B., Oustric, S., et al. 2015. "Off-label Prescribing in Pediatric Outpatients.” Pediatrics 135 (1): 49-58.

[9] Regulation (EC) No $1901 \mathrm{f} 2006$ of Ihc European Parliament and of the Council of 12 December 2006 on medicinal products for paediatric use [online]. Available from http://eur-lex.europa.eu/legal-content/EN/TXT/PDF/?uri= CELEX:32006R1901\&from=en

[10] Bellis, J. R., Kirkham, J. J., Nunn, A. J., and Pirmohamed, M. 2014 "Adverse Drug Reactions and Off-label and Unlicensed Medicines in Children: a Prospective Cohort Study of Unplanned Admissions to a Paediatric Hospital.” Br J Clin Pharmacol 77 (3): 545-53.

[11] Thiesen, S., Conroy, E. J., Bellis, J. R., Bracken, L. E., Mannix, H. L., Bird, K. A., et al. 2013. "Incidence, Characteristics and Risk Factors of Adverse Drug REactions in Hospitalized Children-a Prospective Observational Cohort Study of 6,601 Admissions.” BMC Med 11: 237.

[12] Aagaard, L., and Hansen, E. H. 2014. “Adverse Drug Reactions Associated with Asthma Medications in Children: Systematic Review of Clinical Trials.” Int J Clin Pharm 36 (2): 243-52.

[13] Aagaard, L., and Hansen, E. H. 2014. “Adverse Drug Reactions in Children Reported by European Consumers from 2007 to 2011.” Int J Clin Pharm 36 (2): 295-302.

[14] Choonara, I. 2012. "Educational Paper: Aspects of Clinical Pharmacology in Children Pharmacovigilance and Safety.” European Journal of Pediatrics 172 (5): 577-80.

[15] D’Amore, C., Menniti-Ippolto, F., and Traversa, G. 2014. "La Farmacovigilanza in età Pediatrica." Prospettive in Pediatria 44 (175): 203-7.

[16] Ferrajolo, C., Capuano, A., Trifirò, G., Moretti, U., Rossi, F., and Santuccio, C. 2014. "Pediatric Drug Safety Surveillance in Italian Pharmacovigilance Network: an Overview of Adverse Drug Reactions in the Years 2001-2012.” Expert Opin Drug Saf 13 (1): S9-20.

[17] Fabiano, V., Mameli, C., Cattaneo, D., Delle Fave, A., Preziosa, A., Mele, G., et al. 2012. "Perceptions and Patterns of Use of Generic Drugs Among Italian Family Pediatricians: First Round Results of a Web Survey.” Health Policy 104: 247-52.

[18] ENCEPP- EU PAS Register. ENCEPP STUDY SEAL 2016. Napoleone, E.: "REDS (REspiratory Drugs Survey) STUDY: Active surveillance of respiratory drugs, especially Inhaled Steroids (IS) in children”. Accessed Jenuary 29, 2016 http://www.encepp.eu/encepp/viewResource.htm?id=174 52

[19] Pellegrino, P., Carnovale, C., Cattaneo, D., Perrone V., Antoniazz, S., Pozzi, M., et al. 2013. "Pharmacovigilance Knowledge in Family Paediatricians: A Survey Study in Italy.” Health Policy 113 (1-2): 216-20.

[20] Napoleone, E., and Scasserra, C. 2015. "Pharmacovigilance in Pediatric Age: The Role of FP-MCRN (Family Pediatricians-Medicines for Children Research Network).” J Pharmacovigilance 3: 168.

[21] Napoleone, E., Lavalle, A., Scasserra, C., and Ricci, M., 2016. "Active Surveillance on the Use of Antibiotics in Children, Particularly in the Age Group from 0 to 2 Years.” J Pharmacovigil 4: 205.

[22] Napoleone, E. 2016. “The Appropriate Use of Antibiotics in Early Children: the FP-MCRN Study.” J Develop Drugs 5 (2): 87.

[23] Napoleone, E., Lavalle, A., Scasserra, C., Gentile, S., and Ricci, M. 2016. "Improper Use of Antibiotics in Early Children Increases the risk of ADRs and Drug Resistance: the FP-MCRN Study.” Cogent Medicine 3: 1265203, 3-4. 